\title{
BMJ Open Community-based participatory research to improve life quality and clinical outcomes of patients with breast cancer (DianaWeb in Umbria pilot study)
}

To cite: Villarini M, Lanari $\mathrm{C}$, Nucci D, et al. Communitybased participatory research to improve life quality and clinical outcomes of patients with breast cancer (DianaWeb in Umbria pilot study). BMJ Open 2016;6:e009707. doi:10.1136/bmjopen-2015009707

- Prepublication history for this paper is available online. To view these files please visit the journal online (http://dx.doi.org/10.1136/ bmjopen-2015-009707).

Received 12 August 2015 Revised 2 March 2016 Accepted 14 April 2016

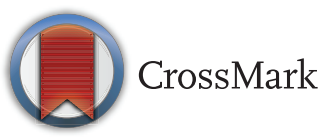

For numbered affiliations see end of article.

Correspondence to Professor Massimo Moretti; massimo.moretti@unipg.it and Dr. Anna Villarini; anna. villarini@istitutotumori.mi.it

\section{ABSTRACT}

Introduction: Breast cancer (BC) is the most frequent cancer in Europe and the International Agency for Research on Cancer (IARC) has estimated over 460000 incident cases per year. Survival among patients with $B C$ has increased in the past decades and EUROCARE-5 has estimated a 5-year relative survival rate of $82 \%$ for patients diagnosed in 2000-2007. There is growing evidence that lifestyle (such as a diet based on Mediterranean principles associated with moderate physical activity) may influence prognosis of $\mathrm{BC}$; however, this information is not currently available to patients and is not considered in oncology protocols. Only a few epidemiological studies have investigated the role of diet in $\mathrm{BC}$ recurrence and metastasis.

Methods and analysis: DianaWeb is a communitybased participatory research dedicated to patients with $\mathrm{BC}$ and represents a collaborative effort between participants and research institutions to determine if specified changes in lifestyle would result in improved outcomes in terms of quality of life or survival. The aim of the study is to recruit a large number of participants, to monitor their lifestyle and health status over time, to provide them tips to encourage sustainable lifestyle changes, to analyse clinical outcomes as a function of baseline risk factors and subsequent changes, and to share with patients methodologies and results. DianaWeb uses a specific interactive website (http://www.dianaweb.org/) and, with very few exceptions, all communications will be made through the web. In this paper we describe the pilot study, namely DianaWeb in Umbria.

Ethics and dissemination: DianaWeb does not interfere with prescribed oncological treatments; rather, it recommends that participants should follow the received prescriptions. The results will be used to plan guidelines for nutrition and physical activity for patients with BC. The pilot study was approved by the ethics committee of the University of Perugia (reference number 2015-002), and is supported by Fondazione Cassa di Risparmio di Perugia (2013.0185 021).

\section{INTRODUCTION}

Breast cancer (BC) is the most frequent cancer among the European population. The International Agency for Research on Cancer (IARC) estimated a number of $464000 \mathrm{BC}$ incident cases per year in Europe, more than colorectal cancer $(447000)$, prostate cancer (417000) and lung cancer (410 000). In Italy, the annual age-standardised BC incidence is $118 / 100000$ and it is still increasing. ${ }^{1}$

Patients' survival increased in the past decades and the prevalence of women with previous $\mathrm{BC}$ experience is rocketing, being presently of the order of 4 million in Europe and 500000 in Italy. The EUROCARE-5 project estimated that 5 -year relative survival of European patients with BC (ie, survival in absence of non-BC deaths) was $82 \%$ for patients diagnosed in 2000-2007. ${ }^{2}$

Investigating whether lifestyle factors affect survival of patients with BC is a relatively new area of research, but there is growing evidence that the effect of lifestyle may be of the same order of magnitude as the effect of the usual clinicopathological risk factors (tumour node metastasis (TNM) classification, hormonal receptors expression, Ki67). In fact, epidemiological studies have shown that the occurrence and prognosis of $\mathrm{BC}$ are affected by modifiable lifestyle factors and biomarkers such as obesity, metabolic syndrome (conventionally defined by the presence of 3 or more of the following risk factors: abdominal obesity, hypertension, hyperglycaemia, high triglycerides or low high-density lipoprotein cholesterol (HDL-C)), sedentary lifestyle, high fat intake, alcohol consumption, high fasting glucose plasma levels, subclinical chronic inflammation, high plasma insulin 
and sex hormone levels, low sex hormone-binding protein. $^{34}$

Obesity is an independent risk factor for postmenopausal BC and, in particular, for estrogen receptor (ER)-positive/progesterone receptor (PR)-positive BC. Moreover, obesity is associated with worse prognosis, before and after menopause. ${ }^{5}$ It is also related to poor quality of life and increased risk of developing comorbid conditions. ${ }^{6}$ Both chemotherapy and, to a lesser extent, tamoxifen and non-steroidal aromatase inhibitors have been associated with weight gain. ${ }^{7}$ Moreover, weight gain during and after cancer treatment has been reported to increase the risk of recurrences. ${ }^{8}$

Among the potential mechanisms behind the association between weight gain, obesity, and recurrence and mortality of $\mathrm{BC}$, there is an increased conversion of androgens to oestrogens in peripheral adipose tissue and increased circulating levels of insulin, insulin-like growth factors (IGFs) and leptins, which promote tumour cell proliferation. ${ }^{9}$

It has been shown that high fasting glycaemia, even within the normal range, is associated with a significantly increased risk of $\mathrm{BC}^{10} 11$ and recently that, in patients with $\mathrm{BC}$, high glycaemic levels are associated with higher incidence of recurrences. $^{12} 13$

Also, metabolic syndrome is associated with increased risk of $\mathrm{BC}$ in postmenopausal women, ${ }^{14}$ and with $\mathrm{BC}$ recurrences in patients diagnosed before and after menopause. ${ }^{15}$ After adjustment for tumour stage and hormonal receptors expression, patients with $\mathrm{BC}$ with metabolic syndrome have a 2.2-fold higher risk of developing distant metastasis than patients without any metabolic syndrome trait. Metabolic syndrome is usually associated with high insulin and testosterone levels, and with subclinical chronic inflammation. Independently of the presence of metabolic syndrome, several studies have shown that high plasma testosterone levels are associated with $\mathrm{BC}$ incidence ${ }^{16}$ and that patients with $\mathrm{BC}$ with high testosterone levels have the worst prognosis. ${ }^{17} 18$ Insulin stimulates the synthesis of testosterone in the ovary, and high plasma insulin levels are associated with increased $\mathrm{BC}$ incidence ${ }^{19}$ and increased risk of recurrences. ${ }^{20}$ Insulin also increases the bioavailability of IGF-I (through the promotion of its liver synthesis and the inhibition of the synthesis of its binding proteins IGFBP1 and IGFBP2), and bioavailable IGF-1 is associated with BC incidence $^{21}$ and prognosis. ${ }^{22}$ Also, high plasma levels of $\mathrm{C}$ reactive protein (CRP), even within the normal range, are associated with increased incidence, ${ }^{23}$ increased risk of recurrence ${ }^{24-26}$ and shorter survival ${ }^{27}$ in patients with advanced BC.

All these factors can be modified favourably through adopting a diet inspired to the traditional Mediterranean Diet and to the European Code Against Cancer (ECAC; http://cancer-code-europe.iarc.fr), the latter including recommendations on diet and on physical exercise of moderate intensity. The ECAC recommends eating plenty of whole grain cereals, pulses, vegetables and fruit, to avoid sugared drinks and processed meat, and to limit red meat, alcoholic beverages and calorie-dense food, which is basically the traditional Mediterranean Diet. The analysis of prospective cohort studies suggested that a high score of adherence to the ECAC is associated with a $30-50 \%$ lower incidence of $\mathrm{BC},{ }^{28} 29$ and several studies have suggested a decrease in $\mathrm{BC}$ recurrence and death in patients who exercise at least $30 \mathrm{~min} /$ day. $^{30-32}$

This information is not currently available to patients and is not yet included in oncology protocols. With a few exceptions, physicians are also not aware of these scientific results and are not yet culturally prepared for lifestyle prescriptions.

DianaWeb is a community-based participatory research (CBPR) offered to patients with BC, whatever the clinical stage of their disease. There is increasing interest in CBPR which has been defined by Minkler and Wallerstein ${ }^{33}$ as 'a process that involves community members or recipients of interventions in all phases of the research process'. Therefore, DianaWeb represents a collaborative effort between participants and research institutions to determine if a specified change, for example, in lifestyle, would result in improved outcome, for example, quality of life or survival.

The basic scientific strategy in the DianaWeb study is to recruit a large number of participants, to monitor participants' lifestyle and health status over time, to provide them with evidence-based recommendations and tips for sustainable changes, to analyse clinical outcomes as a function of baseline risk factors and subsequent changes, and to share with them methodologies and results.

DianaWeb will use a specific interactive website, and, with very few exceptions, all communications between participants, and between participants and the research team, will be through the web.

In this paper, we describe the pilot study (namely DianaWeb in Umbria) aimed at recruiting at least 500 patients in Central Italy (Umbria and bordering Regions). The full study is intended to recruit over 50000 patients in Italy.

\section{METHODS}

\section{Study hypothesis and aims}

We hypothesise that an interactive CBPR may increase patients' compliance with lifestyle recommendations (with respect to usual face-to-face or leaflet-based recommendations) and significantly decrease $\mathrm{BC}$ recurrence risk factors and $\mathrm{BC}$ mortality.

Prevention of $\mathrm{BC}$ recurrences with low-cost technologies, easily available to everybody, is a priority for public health and public finances. Informing about the major modifiable risk factors affecting the progression of $\mathrm{BC}$ and metastatic diffusion, and about how to reduce their impact, may also improve prognosis and prolong life in women with advanced disease.

Moreover, by improving the health literacy of patients with $\mathrm{BC}$, we have the aim of reducing the risk they 
become prey of 'alternative' medicines thus declining evidence-based therapy.

The DianaWeb aims are: (1) to build a cohort of women with a previous diagnosis of $\mathrm{BC}$ and be willing to actively participate in the project; (2) to propose a nutritional switch towards a diet that is poor in refined carbohydrates, fats and animal proteins, and rich in unrefined cereals, pulses and other vegetable foods, according to the ECAC guidelines; (3) to promote regular physical activity of moderate intensity; (4) to monitor the change over time of dietary habits, exercise, body weight and the prevalence of metabolic syndrome parameters (including markers of chronic inflammation and of insulin resistance); (5) to monitor the occurrence of new BC-related clinical events and quality of life of the study participants and (6) to test the hypothesis that the participants who succeed in improving their pattern of risk factors can reduce the incidence of cancer recurrences (local, remote or new BC) and increase survival if already affected by metastasis.

\section{Experimental design}

DianaWeb is a CBPR for the prevention of BC recurrences and the improvement of prognosis through lifestyle modifications.

DianaWeb has a prospective, before-after study design. Since the association of metabolic syndrome or its components with cancer development and cancerrelated mortality is always more frequently highlighted in the literature, ${ }^{34}$ the primary outcome of the DianaWeb in Umbria pilot study is the decrease in metabolic syndrome status, and secondary outcomes in the full-scale DianaWeb study include recurrences and mortality.

In the DianaWeb in Umbria pilot project, about 500 Italian women with a diagnosis of $\mathrm{BC}$ agreed to participate. The women could be with or without metastasis, local recurrence or second cancers; with in situ or invasive cancer, whatever the disease stage at diagnosis; whatever histological diagnosis; whatever the time elapsed since diagnosis. All the above traits will be taken into account as confounders in the statistical analysis.

The basic scientific strategy is to register the clinical history of the patients, provide them with evidence-based recommendations and tips for sustainable lifestyle changes, monitor participant changes, and analyse their effects on clinical outcome.

Participants will be requested to provide clinical and pathological information on their disease at the time of diagnosis and at periodic clinical follow-ups. Enrolled women will be provided with up-to-date evidence-based scientific information about the lifestyle that may prevent recurrences and cancer progression. Every few months, they will receive short questionnaires on their lifestyle, health status and treatments.

The scientific analysis will focus on the association of baseline dietary habits, physical exercise, biomarkers and anthropometric data, and of their changes over time, with clinical outcomes. Women will have access to their individual row data and to anonymised data on the whole group of participants. In the DianaWeb interactive website, individual information is protected but elaborated information (eg, descriptive statistics, case-control and cohort analyses) is open to all participants.

DianaWeb requires the following steps:

1. Setting up of a special interactive website for keeping in contact with recruited women;

2. Dissemination of the project to the public (press releases, public conferences, flyers to be distributed through pharmacies, doctors, cancer centres, charities, etc). On the basis of previous experience, word of mouth among patients who meet during clinical controls is expected to be the most effective recruitment strategy;

3. Recruitment: patients will be enrolled on a voluntary basis, will sign an informed consent form, will fill in a questionnaire on lifestyle, nutrition and medical history, and will provide anthropometric data (weight, height, waist circumference, blood pressure (BP)), results of routine biochemical analysis, and clinical information (histology report and hospital discharge letters, any other subsequent diagnosis);

4. Intervention to promote improved nutrition and physical activity through videos and lectures available on the website (theoretical lectures on preventive strategies, and practical videos on cooking techniques and specific physical exercises), and applications for smartphone; we will develop specific recommendations for women with different characteristics (lean, overweight, obese, active/sedentary lifestyle, dysmetabolic patients, patients with mutations in breast cancer genes (ie, BRCA1 and BRCA2), patients under chemotherapy, hormonal therapy or radiotherapy, patients suffering from joint pain, osteoporosis, menopausal symptoms, cardiovascular toxicity and other symptoms related to the side effects of treatments);

5. Monitoring of lifestyle changes, health changes and quality of life through periodic questionnaires; the pilot study will be completed in late 2016 .

The scientific method of the DianaWeb study includes three phases. The first phase started in January 2015 and closed in June 2015. During the first phase, we created an interactive website. The home page contains a summary of the project in plain language and instructions on how to participate in phase II of the project. During phase II, we will ask the patients interested in joining the project to fill in a personal data sheet with valid email address and a mobile phone number. In this phase, we will send by email to all participating patients an information consent (IC) form and detailed explanation about the project. Once the signed IC (by mail or certified email) with a copy of the identity card, hospital discharge letter reporting the diagnosis of $\mathrm{BC}$ and the histology report are received, we will send it back to the patient, in order to allow her to access the project, 
credentials for login (ie, identification (ID) and password $(\mathrm{PW}))$. Once the patient accesses with her ID and PW, a personal DianaWeb reference code is assigned and automatically reported in the patient's set of forms. The PDF file of the signed IC will be stored in a protected folder, whereas the paper sheets are stored in a locked cabinet. Clinical data (TNM, grading, receptor (ER, PR, ErbB2), p53, Ki67, date of the first surgery, type of treatment, date and type of recurrences) will be entered into the database. In phase III, once the patient has completed the previous steps, she will be notified that she is enrolled in the DianaWeb study and invited to open her personal information section of the website. After the patient has joined the study can access the web pages containing the forms she is requested to fill in: (1) anthropometry form (with instructions on how to make the measurements at home of height, weight, waist circumference, BP), (2) medical history questionnaire, (3) 24 hours recall on food intake and physical activity, (4) short questionnaire on Mediterranean diet and (5) results of the last routine blood tests (glycaemia, cholesterol tot, HDL-C, low-density lipoprotein cholesterol (LDL-C), triglycerides, CRP, liver tests, plasma albumin, vitamin $\mathrm{D}$ ). The forms have to be completed within the first month since recruitment. Insulin and testosterone are not routinely evaluated on blood samples of patients with $\mathrm{BC}$, therefore these analyses will be specifically required for patients recruited in the DianaWeb study.

The 24 hours recall sheet contains a list of 65 food items. The participants are asked to report whether the listed foods were consumed the previous day (serving size and weight, and ricipes of food preparations are not specified). The recall sheet also contains five questions about the time spent on physical exercise on the day before the interview and enquiring on work and recreational physical activity duration (hours/minutes) and intensity (moderate, vigorous). The 24 hours recall sheet was developed to assess dietary habits in the Diana-5 trial and was used to calculate a score of adherence to the World Cancer Research Fund/American Institute for Cancer Research (WCRF/AICR) recommendations. Analysis of data obtained with 24 hours recall in the Diana-5 study has suggested that compliance with the WCRF/AICR recommendations may reduce the risk of cancer and other chronic diseases through the reduction of metabolic syndrome. ${ }^{35} 36$

A 24-point food-items questionnaire, an adaptation of the previously validated MEDAS (a 14-points Mediterranean Diet Adherence Screener) ${ }^{37}$ is aimed at assessing adherence to the traditional Mediterranean diet. The 24-items screener includes 12 additional items that are critical to evaluate the intake of whole grains and/or legumes, non-starchy vegetables, processed meat, milk and dairy products.

From the second month onwards, participants will be asked to post regularly, every 3 months, a 24 hours recall on food frequency and physical exercise. Periodically, every 12 months, to all women enrolled in the study, we will send a query about health status (any changes about your state of health? yes/no). If the answer is yes, a message will appear asking the patient to send by mail or certified email the documentation on the diagnosis. Each year, the patient will receive an email asking her to update anthropometry (with attached instructions to make the measurements at home of height, weight, waist circumference, BP) and new chemical chemistry data (if any). An alert will remind the patient to complete the forms in case of missing information and a smiling face will appear each time the patient will succeed. Every month, the system will email the patient with kitchen recipes, prevention tips or reminders on physical activity.

To allow the patient to contact us, a section for questions and proposals has been created with buttons to indicate with whom the patient wants to interact (principal investigator, administrator, medical doctor, nutritionist, biologist, exercise specialist), or the other participating women. The maximum number of characters allowed is 500. The frequently asked questions (FAQs) section has been created in the home page to be progressively implemented. When the patient completes all the steps, she will receive a free phone number for emergencies, active 2 hours a day for 5 days a week.

During this phase, we will encourage DianaWeb participants to organise meetings, conferences, kitchen classes, walking groups, exchange of information on recipes and on their strategy to comply with the recommendations. The DianaWeb staff will participate in these activities.

The basic lifestyle recommendations are those in the ECAC. ${ }^{38-41}$ For overweight patients, we will stress the evidence-based recommendations for losing weight: reduce protein intake, ${ }^{42}$ avoid chips, potatoes, processed meat, red meat, sugared beverages, white bread, commercial pastries, refined flour. ${ }^{43}$ We will teach how to cook whole grain cereals, legumes, seeds, vegetables and low glycaemic index desserts, which have proven to help losing weight. ${ }^{44}$ Specific dietary and exercise counselling will be offered to patients with hypertension, hyperglycaemia, diabetes, dyslipidaemia, heart diseases, osteoporosis, liver steatosis and chronic inflammation. ${ }^{45-47}$

\section{Feasibility}

The primary purposes of the DianaWeb in Umbria pilot study are to guarantee that the study implementation is practical and to reduce threats to the validity of the study's outcomes. The aspects of feasibility that will be examined with the DianaWeb in Umbria pilot study are: (1) number of enrolled patients; (2) refusal rates for participation after recruitment, retention and follow-up rates as the participants move through the study and (3) adherence rates to study procedures, intervention attendance and engagement. We will also evaluate if eligibility criteria are clear, if the participants are able to understand the questions and the data collection methods, if they respond with missing or unusable data. Regarding data collection, we will assess if the participants have enough time and are able to 
complete data collection procedures and if the overall data collection plan involves a reasonable amount of time, or if it creates a burden for the participants. Furthermore, we will estimate the relevance and affordability of the intervention to obtain significant change.

\section{Statistical power}

In the ongoing Diana-5 study, a randomised controlled trial aimed at evaluating the effectiveness of diet modification in reducing BC recurrences, ${ }^{36}$ we have observed a marked improvement in metabolic parameters associated with cancer progression in the intervention group (ie, patients who participated monthly in kitchen classes and physical exercise sessions). Moreover, we have also observed a significant reduction in several metabolic syndrome parameters (ie, body weight, waist circumference, serum cholesterol and triglycerides) in patients enrolled in the control group who have received only written lifestyle recommendations (unpublished data), the same that we are proposing in the DianaWeb study. On this basis, we have estimated that in the DianaWeb in Umbria pilot study we would have over $90 \%$ power of obtaining significant differences in the considered metabolic parameters between compliant and non-compliant women, with a target sample of 500 patients.

In the full-scale study, we expect to reach a sufficient number of patients to eventually test the prognostic effect of lifestyle and modification of lifestyle both in the early and advanced stages of disease, and in relevant subgroups of 1-2000 patients (eg, triple negative BC), with statistical power of the order of $85-95 \%$ for a $25-33 \%$ risk reduction in the upper quintile of compliance.

\section{Ethics}

DianaWeb does not interfere with prescribed oncological treatments; rather, it recommends participants to follow the prescriptions that they have received. The participants themselves, however, may exchange information on the treatment that they have received, and this may stimulate patients to become more critically aware of their health decisions and to request a second opinion.

\section{Potential biases and limits}

Given that in the DianaWeb study the greater part of the contact with patients will be through the website, the study design could be prone to high dropout rates of participants.

Another limitation is that we will use a food and physical activity 24 hours recall that depends on the respondent's full cooperation. However, diet and physical activity history will be taken regularly every 3 months and, as such, should give more valid estimates of patients' habits.

Finally, since participation is limited to women who have an email address, our findings may not necessarily reflect the overall situation of $\mathrm{BC}$ survivors. In fact, the inability to use internet might reduce the participation of older patients. However, there is no reason to suppose that this aspect could bias the results of those women who will be able to participate.

\section{CONCLUSIONS}

At most, the institutional studies on BC prognosis in relation to lifestyle have recruited about 2000 women. The DianaWeb study is aimed at following a greater population. A collaborative effort between patients and research institutions to engage in researches that benefit the community is a central tenet of CBPR.

The aim of the DianaWeb study is to improve (both in patients and in researchers) the framework of knowledge about risk factors associated with $\mathrm{BC}$ recurrence and survival using a specific interactive website. In particular, we intend to verify whether sustainable lifestyle changes related to nutrition and physical activity can significantly reduce important risk factors for recurrence (such as the factors characterising the metabolic syndrome) and ultimately the incidence of recurrence of $\mathrm{BC}$ and secondary tumours. The results obtained will be used to plan guidelines for nutrition and physical activity for patients with BC.

The cost of cancer care in Italy, was about $€ 7.5$ billion in 2009, thus representing $6.7 \%$ of total health expenditure and appears to be increased. Every year in Italy, $>10000$ patients develop recurrence or metastasis and about 39500 women develop BC; 43000 new cases of BC will be estimated to occur in 2020, with increased costs of $8.7 \%$ compared with the total expenditure in 2009 in Italy.

A prevention approach using an easily accessible web site and low-cost technology is an achievable social challenge, significant both for public health and for our economy.

\section{Author affiliations}

${ }^{1}$ Department of Pharmaceutical Sciences, University of Perugia, Perugia, Italy

${ }^{2}$ Department of Experimental Medicine, University of Perugia, Perugia, Italy

${ }^{3}$ School of Specialization in Hygiene and Preventive Medicine, University of

Perugia, Perugia, Italy

${ }^{4}$ Associazione "La Grande Via", Brescia, Italy

${ }^{5}$ Department of Preventive and Predictive Medicine, Fondazione IRCCS Istituto Nazionale dei Tumori, Milan, Italy

${ }^{6}$ Department of Biomedical Sciences for Health, University of Milan, Milan, Italy

Acknowledgements The authors would like to thank the study sponsor 'Lega Vita e Salute onlus' for its generous financial support.

Contributors $\mathrm{AV}, \mathrm{FB}$ and $\mathrm{MV}$ conceived the project and procured the project funding; $\mathrm{AV}$ is leading the coordination of the trial; MV is the coordinator of the pilot study. CL, DN, VG, TM, GG and MM assisted with the protocol design. TM, $\mathrm{CL}, \mathrm{DN}, \mathrm{VG}, \mathrm{AB}, \mathrm{EB}$ and $\mathrm{GG}$ are managing the trial including data collection. MV and $A V$ drafted the manuscript. MM and FB helped to draft and extensively revise the manuscript. All authors have approved the final manuscript and are willing to take responsibility for appropriate portions of the content.

Funding This work was supported by Fondazione Cassa di Risparmio di Perugia (grant number 2013.0185 021).

Competing interests None declared.

Patient consent Obtained.

Ethics approval The DianaWeb in Umbria study has received ethical approval from the Local Ethics Committee of the University of Perugia (Comitato Universitario di Bioetica), reference number 2015-002. 
Provenance and peer review Not commissioned; externally peer reviewed.

Open Access This is an Open Access article distributed in accordance with the Creative Commons Attribution Non Commercial (CC BY-NC 4.0) license, which permits others to distribute, remix, adapt, build upon this work noncommercially, and license their derivative works on different terms, provided the original work is properly cited and the use is non-commercial. See: http:// creativecommons.org/licenses/by-nc/4.0/

\section{REFERENCES}

1. Ferlay J, Steliarova-Foucher E, Lortet-Tieulent J, et al. Cancer incidence and mortality patterns in Europe: estimates for 40 countries in 2012. Eur J Cancer 2013:49:1374-403.

2. De Angelis R, Sant M, Coleman MP, et al. Cancer survival in Europe 1999-2007 by country and age: results of EUROCARE-5-a population-based study. Lancet Oncol 2014;15:23-34.

3. Berrino F, Villarini A, De Petris M, et al. Adjuvant diet to improve hormonal and metabolic factors affecting breast cancer prognosis. Ann N Y Acad Sci 2006;1089:110-18.

4. Ghose A, Kundu R, Toumeh A, et al. A review of obesity, insulin resistance, and the role of exercise in breast cancer patients. Nutr Cancer 2015;67:197-202.

5. Chan DS, Norat T. Obesity and breast cancer: not only a risk factor of the disease. Curr Treat Options Oncol 2015;16:22.

6. Rock CL, Demark-Wahnefried W. Nutrition and survival after the diagnosis of breast cancer: a review of the evidence. $J$ Clin Oncol 2002;20:3302-16.

7. Makari-Judson G, Braun B, Jerry DJ, et al. Weight gain following breast cancer diagnosis: implication and proposed mechanisms. World J Clin Oncol 2014;5:272-82.

8. Villarini $A$, Pasanisi $P$, Raimondi $M$, et al. Preventing weight gain during adjuvant chemotherapy for breast cancer: a dietary intervention study. Breast Cancer Res Treat 2012;135:581-9.

9. Carmichael AR. Obesity and prognosis of breast cancer. Obes Rev 2006; 7:333-40.

10. Muti $P$, Quattrin T, Grant BJ, et al. Fasting glucose is a risk factor for breast cancer: a prospective study. Cancer Epidemiol Biomarkers Prev 2002;11:1361-8.

11. Sieri S, Muti P, Claudia A, et al. Prospective study on the role of glucose metabolism in breast cancer occurrence. Int $J$ Cancer 2012;130:921-9.

12. Contiero P, Berrino F, Tagliabue G, et al. Fasting blood glucose and long-term prognosis of non-metastatic breast cancer: a cohort study. Breast Cancer Res Treat 2013;138:951-9.

13. Minicozzi $P$, Berrino F, Sebastiani $F$, et al. High fasting blood glucose and obesity significantly and independently increase risk of breast cancer death in hormone receptor-positive disease. Eur $J$ Cancer 2013;49:3881-8.

14. Agnoli C, Berrino F, Abagnato CA, et al. Metabolic syndrome and postmenopausal breast cancer in the ORDET cohort: a nested case-control study. Nutr Metab Cardiovasc Dis 2010;20:41-8.

15. Berrino F, Villarini A, Traina A, et al. Metabolic syndrome and breast cancer prognosis. Breast Cancer Res Treat 2014;147:159-65.

16. Key T, Appleby $\mathrm{P}$, Barnes I, et al. Endogenous sex hormones and breast cancer in postmenopausal women: reanalysis of nine prospective studies. J Natl Cancer Inst 2002;94:606-16.

17. Berrino F, Pasanisi $P$, Bellati $C$, et al. Serum testosterone levels and breast cancer recurrence. Int $J$ Cancer 2005;113:499-502.

18. Micheli A, Meneghini E, Secreto $G$, et al. Plasma testosterone and prognosis of postmenopausal breast cancer patients. J Clin Oncol 2007;25:2685-90.

19. Gunter MJ, Hoover DR, Yu H, et al. Insulin, insulin-like growth factor-I, and risk of breast cancer in postmenopausal women. $J$ Natl Cancer Inst 2009;101:48-60.

20. Goodwin PJ, Ennis M, Pritchard KI, et al. Fasting insulin and outcome in early-stage breast cancer: results of a prospective cohort study. J Clin Oncol 2002;20:42-51.

21. Key TJ, Appleby PN, Reeves GK, et al, Endogenous H, Breast Cancer Collaborative G. Insulin-like growth factor 1 (IGF1), IGF binding protein 3 (IGFBP3), and breast cancer risk: pooled individual data analysis of 17 prospective studies. Lancet Oncol 2010;11:530-42.

22. Pasanisi $\mathrm{P}$, Berrino F, De Petris M, et al. Metabolic syndrome as a prognostic factor for breast cancer recurrences. Int $\mathrm{J}$ Cancer 2006;119:236-8.
23. Siemes C, Visser LE, Coebergh JW, et al. C-reactive protein levels, variation in the C-reactive protein gene, and cancer risk: the Rotterdam Study. J Clin Oncol 2006;24:5216-22.

24. Pierce BL, Ballard-Barbash R, Bernstein L, et al. Elevated biomarkers of inflammation are associated with reduced survival among breast cancer patients. J Clin Oncol 2009;27:3437-44.

25. Allin $\mathrm{KH}$, Nordestgaard $\mathrm{BG}$, Flyger $\mathrm{H}$, et al. Elevated pre-treatment levels of plasma C-reactive protein are associated with poor prognosis after breast cancer: a cohort study. Breast Cancer Res 2011;13:R55.

26. Villaseñor A, Flatt SW, Marinac C, et al. Postdiagnosis C-reactive protein and breast cancer survivorship: findings from the WHEL study. Cancer Epidemiol Biomarkers Prev 2014;23:189-99.

27. Algire $\mathrm{C}$, Amrein L, Bazile M, et al. Diet and tumor LKB1 expression interact to determine sensitivity to anti-neoplastic effects of metformin in vivo. Oncogene 2011;30:1174-82.

28. Romaguera D, Vergnaud AC, Peeters PH, et al. Is concordance with World Cancer Research Fund/American Institute for Cancer Research guidelines for cancer prevention related to subsequent risk of cancer? Results from the EPIC study. Am J Clin Nutr 2012;96:150-63.

29. Hastert TA, Beresford SA, Patterson RE, et al. Adherence to WCRF/ AICR cancer prevention recommendations and risk of postmenopausal breast cancer. Cancer Epidemiol Biomarkers Prev 2013:22:1498-508.

30. Holick CN, Newcomb PA, Trentham-Dietz A, et al. Physical activity and survival after diagnosis of invasive breast cancer. Cancer Epidemiol Biomarkers Prev 2008;17:379-86.

31. Holmes MD, Chen WY, Hankinson SE, et al. Physical activity's impact on the association of fat and fiber intake with survival after breast cancer. Am J Epidemiol 2009;170:1250-6.

32. Zhong $\mathrm{S}$, Jiang $\mathrm{T}, \mathrm{Ma} \mathrm{T}$, et al. Association between physical activity and mortality in breast cancer: a meta-analysis of cohort studies. Eur J Epidemiol 2014;29:391-404.

33. Minkler M, Wallerstein N. Community-based participatory research for health: from process to outcomes. John Wiley \& Sons, New York. 2nd edn, 2010.

34. Uzunlulu M, Telci Caklili O, Oguz A. Association between metabolic syndrome and cancer. Ann Nutr Metab 2016;68:173-9.

35. Bruno E, Gargano G, Villarini A, et al. Adherence to WCRF/AICR cancer prevention recommendations and metabolic syndrome in breast cancer patients. Int J Cancer 2016;138:237-44.

36. Villarini A, Pasanisi P, Traina A, et al. Lifestyle and breast cancer recurrences: the DIANA-5 trial. Tumori 2012;98:1-18.

37. Schröder H, Mendez MA, Ribas-Barba L, et al. Mediterranean diet and waist circumference in a representative national sample of young Spaniards. Int J Pediatr Obes 2010;5:516-19.

38. Anderson AS, Key TJ, Norat T, et al. European Code Against Cancer 4th edition: obesity, body fatness and cancer. Cancer Epidemiol 2015;39(Suppl 1):S34-45.

39. Norat T, Scoccianti C, Boutron-Ruault MC, et al. European Code Against Cancer 4th edition: diet and cancer. Cancer Epidemiol 2015;39(Suppl 1):S56-66.

40. Leitzmann M, Powers H, Anderson AS, et al. European Code Against Cancer 4th edition: physical activity and cancer. Cancer Epidemiol 2015;39(Suppl 1):S46-55.

41. Scoccianti C, Cecchini M, Anderson AS, et al. European Code Against Cancer 4th Edition: alcohol drinking and cancer. Cancer Epidemiol 2015;39(Suppl 1):S67-74

42. Vergnaud AC, Norat T, Romaguera $D$, et al. Fruit and vegetable consumption and prospective weight change in participants of the European Prospective Investigation into Cancer and Nutrition-Physical Activity, Nutrition, Alcohol, Cessation of Smoking, Eating Out of Home, and Obesity study. Am J Clin Nutr 2012;95:184-93.

43. Mozaffarian D, Hao T, Rimm EB, et al. Changes in diet and lifestyle and long-term weight gain in women and men. $N$ Engl J Med 2011;364:2392-404.

44. Berrino F, Bellati C, Secreto G, et al. Reducing bioavailable sex hormones through a comprehensive change in diet: the diet and androgens (DIANA) randomized trial. Cancer Epidemiol Biomarkers Prev 2001:10:25-33.

45. Ahmed SA, Hamed MA, Omar OS. Clinical utility of certain biomarkers as predictors of breast cancer with or without metastasis among Egyptian females. Tumour Biol 2015;36:815-22.

46. Yigit E, Gönüllü G, Yücel I, et al. Relation between hemostatic parameters and prognostic/predictive factors in breast cancer. Eur $J$ Intern Med 2008;19:602-7.

47. Cheng CW, Adams GB, Perin L, et al. Prolonged fasting reduces IGF-1/PKA to promote hematopoietic-stem-cell-based regeneration and reverse immunosuppression. Cell Stem Cell 2014;14:810-23. 